\title{
Metanephric adenoma with diffuse calcifications: A case report
}

\author{
JINGTAO WU*, QINGQIANG ZHU*, WENRONG ZHU and HONGYING ZHANG \\ Department of Medical Imaging, Subei People's Hospital, Medical School of Yangzhou University, \\ Yangzhou, Jiangsu 225001, P.R. China
}

Received September 2, 2014; Accepted April 29, 2015

DOI: $10.3892 / \mathrm{ol} .2015 .3491$

\begin{abstract}
Metanephric adenoma is a rare and benign renal neoplasm originating in the epithelial cells of the kidney. The tumor has a benign course and a characteristic histopathological appearance, typically exhibiting a solid and poorly-demarcated margin with rare cystic components or calcifications. However, it is often difficult to distinguish metanephric adenoma from malignant neoplasms prior to surgical resection. To the best of our knowledge, only one case of metastasis to the lymph nodes has been described in the literature thus far. The present study retrospectively analyzed one case of surgically and pathologically-confirmed atypical metanephric adenoma. Clinical and pathological analysis, as well as computed tomography scans, revealed a mass with a clearly defined margin and diffuse calcifications. The mass was subsequently resected and the patient recovered well following the procedure.
\end{abstract}

\section{Introduction}

Metanephric adenomas of the kidney are a rare and predominantly benign type of neoplasm that are typically removed by surgical resection (1). The clinical presentation is similar to that of malignant renal masses, and includes polycythemia, hematuria and abdominal pain. Furthermore, metanephric adenomas are occasionally detected incidentally upon imaging (2). Tumors are typically composed of solid, rare cystic components or calcifications with a poorly-defined border (3). To the best of knowledge, thus far, $<200$ total cases of metanephric adenoma and only one case of metastasis to the lymph nodes has been described in the literature (4). Despite the benign nature of this neoplasm, an improved understanding of its pathology may lead

Correspondence to: Dr Jingtao Wu or Dr Qingqiang Zhu, Department of Medical Imaging, Subei People's Hospital, Medical School of Yangzhou University, 98 West Nantong Road, Yangzhou, Jiangsu 225001, P.R. China

E-mail: wujingtaodoctor@163.com

E-mail: zhuqingqiang1983@163.com

*Contributed equally

Key words: metanephric adenoma, diffuse calcifications, atypia to the development of less invasive treatment strategies in the future. The current study presents the case of a male patient diagnosed with atypical metanephric adenoma with diffuse calcifications who subsequently underwent radical nephrectomy.

\section{Case report}

A 23-year-old male patient was admitted to Subei People's Hospital (Yangzhou, China) with a history of flank pain on October 23, 2013, and appeared in good general condition. The patient had no history of occupational exposure to carcinogens.At $>1$ month after the onset of symptoms, a medical examination identified a left renal mass with diffuse calcifications. The mass, including the calcifications, was subsequently resected on October 28, 2013. The patient recovered well following the surgical procedure.

Unenhanced computed tomography (CT) scans were performed using 64-Light speed Ultra (GE Healthcare, Milwaukee, WI, USA), and identified a well-defined mass (diameter, $6.9 \mathrm{~cm}$ ) with diffuse calcifications in the medulla region of the kidney. The attenuation of the solid component of the mass was $22 \mathrm{HU}$ and diffuse calcifications were noted (Fig. 1A). During the cortical phase contrast-enhanced CT scan, attenuation of the solid component demonstrated mild enhancement at a value of less than that in the cortex and medulla regions (36 HU; Fig. 1B). The attenuation of the solid tumor (45 HU) marginally increased during the medullary phase (Fig. 1C). However, during the delayed phase, the attenuation of the tumor decreased to 37 HU (Fig. 1D). Pathological analysis of sections revealed heterogeneous round or ovoid tumor cells arranged in tubules and tubular glands. The tumor cells exhibited little eosinophilic cytoplasm and few cylindrical nuclei, and no karyokinesis was observed by hematoxylin and eosin staining (Fig. 1E). Immunohistochemical analysis (EnVision $^{\mathrm{TM}}$ kit; Dako, Glostrup, Denmark) identified high expression levels of vimentin (Fig. 1F) and pan-cytokeratin (Fig. 1G) (4).

Written informed consent for the present case report was obtained from the patient.

\section{Discussion}

Metanephric adenoma may occur in children and adults, although it is most common in the fifth and sixth decades of life. Furthermore, the disease has a 2:1 female preponderance (5). To the best of our knowledge, only one case of 
A

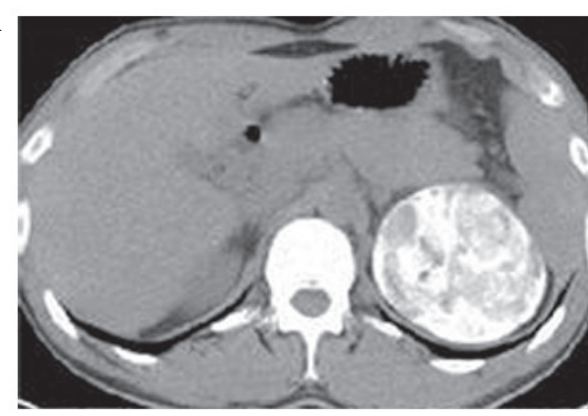

C

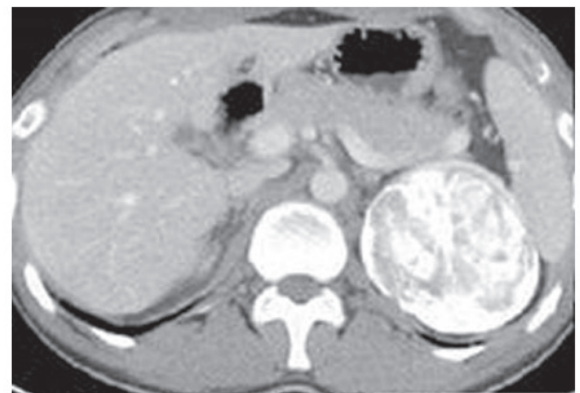

B

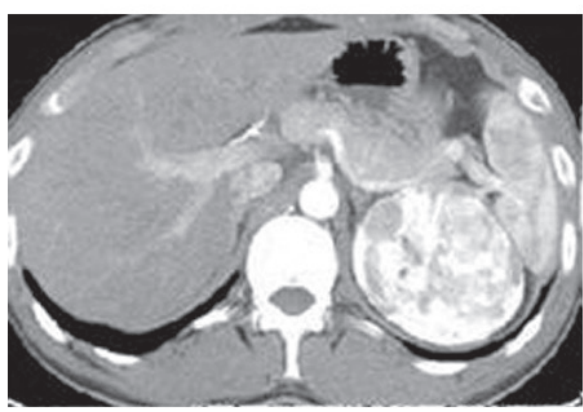

D

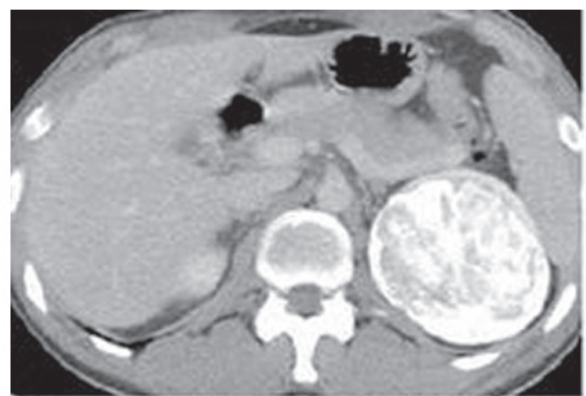

$\mathbf{E}$

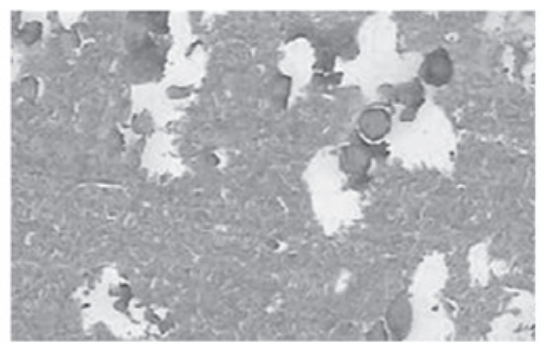

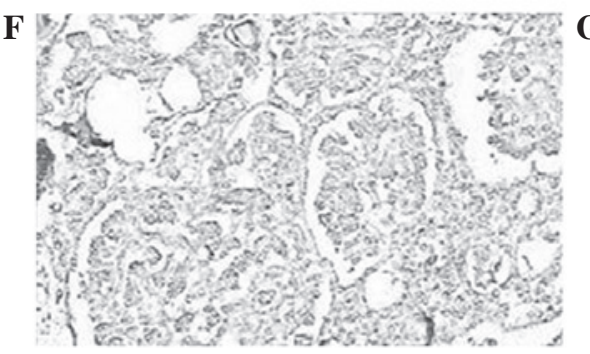

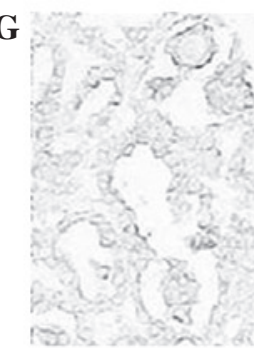

Figure 1. Metanephric adenoma of the left kidney. (A) Unenhanced computed tomography (CT) scan revealing a well-defined mass with diffuse calcifications in the medulla region. The attenuation of the solid section of the mass was $22 \mathrm{HU}$ and diffuse calcifications were noted. (B) Cortical phase contrast-enhanced CT scan, demonstrating mild enhancement of the attenuation of the solid component at a value of less than that in the cortex and medulla (36 HU). (C) Marginally increased attenuation of the solid component of the tumor (45 HU) during the medullary phase and (D) decreased attenuation of the tumor to $37 \mathrm{HU}$ during the delayed phase. (E) Pathological analysis of the tissue sections revealing heterogeneous round or ovoid tumor cells arranged in tubules and tubular glands. The tumor cells exhibited little eosinophilic cytoplasm and few cylindrical nuclei. No karyokinesis was observed upon hematoxylin and eosin staining (magnification, $x 400)$. Immunohistochemical analysis was also performed, revealing high expression levels of (F) vimentin and (G) pan-cytokeratin.

high grade sarcoma arising in association with metanephric adenoma, termed metanephric adenosarcoma, has been reported in the literature thus far (6). Approximately 50\% of cases of metanephric adenoma are incidental findings, however, symptoms include polycythemia, abdominal or flank pain, a mass and hematuria (7). Similarly, presenting symptoms of metanephric adenofibroma include, polycythemia or hematuria while certain cases have been diagnosed from incidental findings (8). Haemorrhagic foci and necrosis are also commonly detected in metanephric adenoma, with calcifications and small cysts present in $\sim 20$ and $10 \%$ of cases, respectively (9).

Previously published studies of metanephric adenoma have described the pathological, clinical and CT imaging features of this rare form of renal carcinoma (10). Tumors typically exhibited solid, rare cystic components or calcifications, a poorly-defined border, and were without retroperitoneal lymph node metastasis (11). Tumors were observed to be centred in the medulla region, with decreased attenuation on unenhanced CT scans and reduced enhancement compared with the adjacent cortex or medulla $(11,12)$. Furthermore, there is typically no evidence of invasion into the renal pelvis or calyx, renal vein, inferior vena cava or ureter (12). However, the current case demonstrated a clearly defined margin with diffuse calcifications, dissimilar to that observed in previous cases. This formation of diffuse calcifications may correlate with intratumoral hemorrhage $(12,13)$. Patients with metanephric adenoma typically have a good prognosis after undergoing total nephrectomy or local resection with kidney preservation (14). Therefore, an accurate diagnosis is important for selecting the most appropriate clinical treatment strategy.

In the present study, the degree of enhancement of the solid component of the metanephric adenoma was lower than that of the normal renal cortex during all enhanced phases. This enhancement pattern is atypical of tumors with a rich blood supply, such as clear cell renal cell carcinoma, renal medullary carcinoma, renal angiomyolipoma and renal angioma $(15,16)$. For example, the degree of enhancement of clear cell renal cell carcinoma is commonly higher than that of the renal cortex (17). These findings indicate that it may be relatively simple to 
distinguish between metanephric adenoma and tumors with a rich blood supply on the basis of differences in enhancement (18). For example, metanephric adenoma, collecting duct carcinaoma, chromophobe renal cell carcinoma and renal lymphoma are hypovascular on enhanced CT imaging compared with renal parenchyma. In addition, medullary involvement and an infiltrative appearance are common findings on CT imaging and may indicate a diagnosis of collecting duct carcinoma (19). The majority of cases of papillary renal cell carcinoma demonstrate marginal enhancement during all enhanced phases; however, papillary renal cell carcinoma may be multifocal and bilateral. In such cases, the tumor typically measures $<2 \mathrm{~cm}$ in diameter at the time of diagnosis and exhibits reduced enhancement.

In conclusion, metanephric adenoma is a rare subtype of renal carcinoma with specific pathological features. Precise diagnosis based on CT imaging findings alone remains difficult, particularly for atypical tumors. Therefore, additional investigation is required in larger patient populations to assist with diagnosis in the future.

\section{References}

1. Küpeli S, Baydar DE, Canakl F, et al: Metanephric adenoma in a 6-year-old child with hemihypertrophy. J Pediatr Hematol Oncol 31: 453-455, 2009.

2. Fan H, Li HZ, Zhang YS, Zhang XB and Shi BB: Clinical character of metanephric adenoma of the kidney: A case report. Beijing Da Xue Xue Bao 45: 654-656, 2013 (In Chinese).

3. Zhu P, Yan F, Yang Z, Meng L and Ao Q: Composite tumor of metanephric adenoma and Wilms' tumor of the kidney: A case report and review of the literature. Oncol Lett 5: 1311-1314, 2013.

4. Mantoan Padilha M, Billis A, Allende D, Zhou M and Magi-Galluzzi C: Metanephric adenoma and solid variant of papillary renal cell carcinoma: Common and distinctive features. Histopathology 62: 941-953, 2013.

5. Blanco LZ Jr, Schein CO, Patel T, et al: Fine-needle aspiration of metanephric adenoma of the kidney with clinical, radiographic and histopathologic correlation: A review. Diagn Cytopathol 41: 742-751, 2013.
6. Masuda A, Kamai T, Mizuno T, et al: Renal metanephric adenoma mimicking papillary renal cell carcinoma on computed tomography: A case report. Urol Int 90: 369-372, 2013.

7. Bastide C, Rambeaud JJ, Bach AM and Russo P: Metanephric adenoma of the kidney: Clinical and radiological study of nine cases. BJU Int 103: 1544-1548, 2009.

8. Moskvina LV, Andreeva IuIu, Frank GA, Alekseev BIu and Petrov AN: Metanephric adenoma of the kidney. Arkh Patol 75: 55-57, 2013 (In Russian).

9. Zambrano N, Vivaldi B and Espinoza R: Metanephric adenoma of the kidney associated with polycythemia and erythromelalgia: Report of one case. Rev Med Chil 140: 629-632, 2012 (In Spanish).

10. Kohashi K, Oda Y, Nakamori M, et al: Multifocal metanephric adenoma in childhood. Pathol Int 59: 49-52, 2009.

11. McNeil JC, Corbett ST, Kuruvilla S and Jones EA: Metanephric adenoma in a five-year-old boy presenting with chyluria: Case report and review of literature. Urology 72: 545-7, 2008

12. Zhu Q, Zhu W, Wu J, Chen W and Wang S: The clinical and CT imaging features of metanephric adenoma. Acta Radiologica 55: 231-238, 2014.

13. Jain M, Rastogi A and Gupta RK: Atypical metanephric adenoma - a case report and review of literature. Int Urol Nephrol 39: 123-127, 2007.

14. Renshaw AA, Freyer DR and Hammers YA: Metastatic metanephric adenoma in a child. Am J Surg Pathol 24: 570-574, 2000.

15. Comerci SC, Levin TL, Ruzal-Shapiro C, et al: Benign adenomatous kidney neoplasms in children with polycythemia: Imaging findings. Radiology 198: 265-268, 1996.

16. Lopez-Beltran A, Scarpelli M, Montironi R and Kirkali Z: 2004 WHO classification of the renal tumors of the adults. Eur Urol 49: 798-805, 2006

17. Drut R, Drut RM and Ortolani C: Metastatic metanephric adenoma with foci of papillary carcinoma in a child: A combined histologic, immunohistochemical, and FISH study. Int J Surg Pathol 9: 241-247, 2001.

18. Amodio JB, Shapiro E, Pinkney L, et al: Metanephric adenoma in an 8-year-old child: Case report and review of the literature. J Pediatr Surg 40: e25-e28, 2005

19. Yoon SK, Nam KJ, Rha SH, et al: Collecting duct carcinoma of the kidney: CT and pathologic correlation. Eur J Radiol 57: 453-460, 2006. 